\title{
Supporting Information by Quantification of the role of chemical desorption in molecular clouds
}

\author{
Adrien Fredon, ${ }^{\dagger}$ Ash K. Radchenko, ${ }^{\dagger}$ and Herma M. Cuppen*,†, \\ $\dagger$ Radboud University Nijmegen, Institute for Molecules and Materials, Heyendaalseweg 135, \\ 6525 AJ Nijmegen, The Netherlands. \\ $\ddagger$ van $t$ Hoff Institute for Molecular Sciences, University of Amsterdam, Science Park 904, \\ 1098 XH Amsterdam, The Netherlands \\ E-mail: h.cuppen@science.ru.nl
}

\section{Termination criteria for dissipation simulations}

The energy dissipation simulations end if one of the seven following outcomes is reached:

1. adsorption: the admolecule remains below $z=40 \AA$ and its total energy is less than a certain threshold value $\left(-0.1 \mathrm{eV}\right.$ for $\mathrm{CO}_{2},-0.4 \mathrm{eV}$ for $\mathrm{H}_{2} \mathrm{O}$, and $0 \mathrm{eV}$ for $\left.\mathrm{CH}_{4}\right)$ for at least 8,6 or $10 \mathrm{ps}$, for $\mathrm{CO}_{2}, \mathrm{H}_{2} \mathrm{O}$ and $\mathrm{CH}_{4}$, respectively.

2. desorption: the admolecule reaches above $z=40 \AA$. The ice surface is roughly located around $z=23 \AA$. With a cutoff of $11 \AA$ the threshold value of $z=40 \AA$ is safe to ensure that the admolecule does not feel the attracting surface.

3. penetration: the admolecule is below $z=20 \AA$ and its velocity vector is pointing downwards.

4. adsorption with $\mathrm{H}_{2} \mathrm{O}$ desorption: the admolecule is classified as adsorbed (outcome 1) and an additional water molecule has desorbed ( $z>40 \AA$ ) ("kick-out" mechanism). 
5. desorption with $\mathrm{H}_{2} \mathrm{O}$ desorption: combination of outcome 2 and kick-out (see outcome 4).

6. penetration with $\mathrm{H}_{2} \mathrm{O}$ desorption: combination of outcome 3 and kick-out.

7. timeout: the default outcome if none of the others is found after 20 ps.

\section{Rotational and vibrational excitations}

This section describes rotational and vibrational excitations in our simulations.

\section{$2.1 \quad$ Rotation}

Over a large set of simulations a fixed amount of rotational energy is randomly distributed over the three (two for $\mathrm{CO}_{2}$ ) rotation axes. This is obtained by diagonalizing the inertia tensor of the admolecule $\mathbf{I}$ to obtain the three eigenvectors $\mathbf{u}_{i}$ and the three associated eigenvalues $u_{i}$ of the inertia tensor. The $\mathbf{u}_{i}$ vectors define the body frame reference $\mathbf{B F}$. A random normal vector $\mathbf{r}$ is generated and projected on the eigenvectors $\mathbf{u}_{i}$ to obtain the amount of energy deposited on the $i^{\text {th }}$ eigenvector

$$
E_{i}=r_{i}^{2} E_{\mathrm{rot}} .
$$

The angular velocity vector $\mathrm{w}^{\mathrm{BF}}$ in the body frame reference can then be obtained through

$$
w_{i}^{\mathrm{BF}}= \pm \sqrt{\frac{2 \times E_{i}}{u_{i}}} .
$$

The \pm sign is chosen randomly. Back conversion to Cartesian coordinates leads to the velocity associated to the rotation for atom $A$

$$
\mathbf{v}_{A}=\mathbf{w}^{\mathrm{CF}} \times \mathbf{r}_{A}^{c o m}
$$


where $\mathbf{r}_{A}^{c o m}$ is the position atom $A$ relative to the center of mass.

\section{$2.2 \quad$ Vibration}

The vibrational excitations are performed by introducing a conformational change in the geometry of the admolecule. We used four different methods to excite the admolecules, which are schematically depicted in Table S1. For type 1, the central atom is displaced which results in both stretching and bending of different internal modes of the admolecule. For the second and third option, only one specific bond is excited instead of a combination of bonds and angles by moving one of the terminal atoms. Terminal atoms that point away from the surface are picked for option 2 and that point towards the surface for option 3. For the fourth and last option, non-symmetric and anti-symmetric excitations are performed. The vibrational energy as a function of the displacement of the moving atom (see Table S1)

is calculated for isolated molecules with a step size of $0.01 \AA$ and then interpolated using a 3rd-order polynomial. Since there is a direct, one-to-one mapping of the displacement to one energy value and vice versa, only four displacements can be used, one for each type, for each vibrational energy value per admolecule and per binding site on the surface. For this reason, the number of different vibrational excitation realizations is limited to forty, instead of the thousands that can be achieved for translational and rotational excitation. Only of translational excitation is used in combination with other excitation types, a large statistics can be obtained. The vibrational excitations induce a structural change in the admolecule which modifies inertia. One should hence excite the vibrational modes before applying rotationally excitation to the admolecule.

\section{Trajectories of water excitation}

The movies show simulation trajectories of a $\mathrm{H}_{2} \mathrm{O}$ admolecule with excitation of either $1 \mathrm{eV}$ of rotational energy, $1 \mathrm{eV}$ of translational energy or a combination of both. In the latter case 
Table S1: Vibrational excitation types. The red arrows indicate what atoms are moving for each type of excitation.

\begin{tabular}{|c|c|c|c|}
\hline Vib type & $\mathrm{CO}_{2}$ & $\mathrm{H}_{2} \mathrm{O}$ & $\mathrm{CH}_{4}$ \\
\hline 1 & $\therefore:{ }_{\mathrm{C}}^{\uparrow}=\stackrel{0}{\mathrm{O}}$ & & \\
\hline 2,3 & $\because \overrightarrow{\mathrm{O}}=\mathrm{C}=\dot{\mathrm{O}}$ & & \\
\hline 4 & $\therefore=\mathrm{C}=\dot{0}$ & & \\
\hline
\end{tabular}

the direction of translation and the axis of rotation is the same as in the pure translation and rotation simulations, respectively. The starting position is also the same in all simulations. The movies have been prepared with the help of VMD. ${ }^{1}$

Movie H2O_T1eV.mp4 excitation of $1 \mathrm{eV}$ of translational energy

Movie H2O_R1eV.mp4 excitation of $1 \mathrm{eV}$ of rotation energy

Movie H2O_T1eV_R1eV.mp4 excitation of $1 \mathrm{eV}$ of translational energy and $1 \mathrm{eV}$ of rotation energy

\section{Changes in astrochemical model}

The following changes are made to code developed by M. Drozdovskaya and C. Walsh.: ${ }^{2,3}$

1. a new treatment of chemical desorption was implemented following Eq. 1 in the main text. For this implementation, the reaction enthalpy for each reaction is required. This is automatically calculated from the individual formation enthalpies of the reactants and products. Gas phase enthalpies are used since computation studies show that the surface has no significant influence on the reaction enthalpies. ${ }^{4,5}$ These are taken from the Active Thermochemical Tables (ATcT) version 1.122g (https://atct.anl. 
gov// ${ }^{6,7}$ or the NIST-JANAF Thermochemical Tables (https://janaf.nist.gov/), ${ }^{8}$ if available. The remaining enthalpies were computed by semi-emperical calculations using MOPAC (PM7,${ }^{9}$ in vacuum at $\left.298 \mathrm{~K}\right) .{ }^{10}$ Although this is not the most accurate method, this is a good quality/computation load balance given the number of species.

2. the frequency or pre-exponential factor in the computation of diffusion, reaction, and thermal desorption rates is taken as the frequency of a free particle $\frac{k T}{h}$ for all molecules. The prefactor was previously

$$
\nu=\sqrt{\frac{2 N_{s} E_{\mathrm{bind}, A}}{\pi^{2} m_{A}}}
$$

where $N_{s}$ is the surface density of binding sites and $m_{A}$ is the mass of species A. Tielens ${ }^{11}$ derived this expression assuming that the vibrational frequency perpendicular to the surface equals the vibrational frequency parallel to the surface and that the binding can be described by a harmonic potential, which might not be an accurate assumption for physisorbed species. It further is derived for desorption and not for reactive events. See further discussion on the prefactor in Ref. ${ }^{12}$

3. implementation of two-product desorption, leading to four different outcomes, with either none, one, or both products returning to the gas phase. Desorption of the products are assumed to be uncorrelated events and can hence be calculated straightforwardly from the individual desorption probabilities:

$$
\begin{aligned}
P_{\text {both desorb }} & =P_{\text {prod1 }}^{\mathrm{CD}} P_{\text {prod2 } 2}^{\mathrm{CD}}, \\
P_{\text {prod1 desorbs }} & =P_{\text {prod1 }}^{\mathrm{CD}}-P_{\text {prod1 }}^{\mathrm{CD}} P_{\text {prod2 } 2}^{\mathrm{CD}}, \\
P_{\text {prod2 desorbs }} & =P_{\mathrm{prod} 2}^{\mathrm{CD}}-P_{\mathrm{prod} 1}^{\mathrm{CD}} P_{\text {prod2} 2}^{\mathrm{CD}} . \\
P_{\text {no desorption }} & =1-\left(P_{\mathrm{prod} 1}^{\mathrm{CD}}+P_{\mathrm{prod} 2}^{\mathrm{CD}}-P_{\mathrm{prod} 1}^{\mathrm{CD}} P_{\text {prod2 }}^{\mathrm{CD}}\right) .
\end{aligned}
$$




\section{References}

(1) Humphrey, W.; Dalke, A.; Schulten, K. VMD: Visual molecular dynamics. J. Mol. Graph. 1996, 14, 33.

(2) Drozdovskaya, M. N.; Walsh, C.; Visser, R.; Harsono, D.; van Dishoeck, E. F. Methanol along the path from envelope to protoplanetary disc. Mon. Not. R. Astron. Soc. 2014, 445,913 .

(3) Drozdovskaya, M. N.; Walsh, C.; Visser, R.; Harsono, D.; van Dishoeck, E. F. The complex chemistry of outflow cavity walls exposed: the case of low-mass protostars. Mon. Not. R. Astron. Soc. 2015, 451, 3836.

(4) Enrique-Romero, J.; Álvarez-barcia, S.; Kolb, F. J.; Rimola, A.; Ceccarelli, C.; Balucani, N.; Meisner, J.; Ugliengo, P.; Lamberts, T.; Kästner, J. Revisiting the reactivity between $\mathrm{HCO}$ and $\mathrm{CH} 3$ on interstellar grain surfaces. Mon. Not. R. Astron. Soc. 2020, 493, 2523.

(5) Koch, D. M.; Toubin, C.; Peslherbe, G. H.; Hynes, J. T. A Theoretical Study of the Formation of the Aminoacetonitrile Precursor of Glycine on Icy Grain Mantles in the Interstellar Medium. J. Phys. Chem. C 2008, 112, 2972-2980.

(6) Ruscic, B.; Pinzon, R. E.; Morton, M. L.; Von laszevski, G.; Bittner, S. J.; Nijsure, K. A., S. G. a nd Amin; Minkoff, M.; Wagner, A. F. Introduction to Active Thermochemical Tables: Several "Key" Enthalpies of Formation Revisited. J. Phys. Chem. A 2004, 108, 9979.

(7) Ruscic, B.; Pinzon, R. E.; Laszewski, G. v.; Kodeboyina, D.; Burcat, A.; Leahy, D.; Montoy, D.; Wagner, A. F. Active Thermochemical Tables: thermochemistry for the 21st century. J. Phys.: Conf. Series 2005, 16, 561. 
(8) Allison, T. JANAF Thermochemical Tables, NIST Standard Reference Database 13. 1996,

(9) Stewart, J. J. P. Optimization of parameters for semiempirical methods V: Modification of NDDO approximations and application to 70 elements. J. Mol. Mode. 2007, 13, 1173.

(10) Stewart, J. J. P. MOPAC2016; 2016.

(11) Tielens, A. G. G. M.; Allamandola, L. J. Composition, structure, and chemistry of interstellar dust. Interstellar Processes. 1987; pp 397-469.

(12) Cuppen, H. M.; Walsh, C.; Lamberts, T.; Semenov, D.; Garrod, R. T.; Penteado, E. M.; Ioppolo, S. Grain Surface Models and Data for Astrochemistry. Space Sci. Rev. 2017, 212, 1. 